\title{
CHIRAL CHANGES OF CORTICAL MICROTUBULE ORIENTATIONS IN EPIDERMIS OF SUNFLOWER HYPOCOTYLS. THE EFFECT OF BLUE AND RED LIGHT
}

\author{
AgATA BURIAN \\ Department of Biophysics and Cell Biology, University of Silesia \\ Jagiellońska 28, 40-032 Katowice, Poland \\ e-mail: aburian@us.edu.pl
}

(Received: September 12, 2006. Accepted: November 6, 2006)

\begin{abstract}
Light and developmental processes affect the cortical microtubule (cMT) orientation. The cMT orientation with a special regard to its chirality was analyzed under the outer epidermal cell walls in different regions of sunflower hypocotyls kept in darkness and after irradiation with blue and red light. The results show that the cMT orientation depends on the cell position along hypocotyl, but generally cMTs are oblique. The oblique orientation has defined chirality: either of Z-form (right-handed) or S-form (left-handed). In the lower region of hypocotyls the Z-form dominates. After irradiation of hypocotyls with blue light this domination has been maintained and appeared also in the upper region. In contrast, after irradiation with red light the Z-form domination has not been apparent. It is proposed that in darkness, variations of cMT orientations in the epidermis along the hypocotyl are due to developmental processes, while blue and red light affect the cMT orientation via "shifting" these processes backward and forward, respectively.
\end{abstract}

KEY WORDS: chirality, Helianthus annuus, cortical microtubules, hypocotyls, epidermis, blue and red light.

\section{INTRODUCTION}

Cytoskeleton plays an important role in plant morphogenesis. The array of cortical microtubules (cMTs), i.e. microtubules underlying the plasma membrane during interphase, is thought to determine the direction of cell growth by influencing the orientation of cellulose microfibrils (Wymer and Lloyd 1996; Baskin 2001). Recently it was shown that localization and guidance of cellulose synthase component (CESA) is spatially and temporally coupled to microtubules (Paredez et al. 2006). However, the relationship between cMTs and cellulose microfibrils is still controversial (Emons et al. 1992; Ketelaar and Emons 2001).

The cMTs are highly dynamic structures. They exhibit periods of growth, pausing and shortening. In Arabidopsis the cMT migration occurs by means of the so called hybrid treadmilling mechanism (Shaw et al. 2003), in which the cMT migration is the net result of polymerization-biased dynamic instability at the leading (plus) ends and slow intermittent depolymerization at the lagging (minus) ends.

In the fluorescence microscopy cMTs are seen as bundles, which in fact consist of overlapping microtubules of mixed polarity (Tian et al. 2004). The cMT bundles are more or less parallel to one another, and are aligned at a particular angle with respect to cell axis. In rapidly elongating cells cMTs are transverse but they shift to oblique and then longitudinal orientation as elongation rate declines (Baskin et al. 1999; Granger and Cyr 2001).

However, even in constant conditions, the orientation of parallel cMTs in epidermal cells is cyclically changing (Mayumi and Shibaoka 1996; Takesue and Shibaoka 1998). This process is called an autonomous reorientation (Hejnowicz 2005). The autonomous reorientation seems to be based on the cMT rotation with variable angular velocity which depends on the orientation itself. In particular the velocity is very high or in other words cMTs are "jumping" when passing either longitudinal or transverse direction, or both of them (Hejnowicz 2005). It is assumed that the external or internal factors affect cMT orientation by changing the velocity of their rotation.

Many external factors, like plant growth regulators (see review by Shibaoka, 1994), electric field (Hush and Overall 1991; Blackman and Overall 1995), mechanical stresses (Fischer and Schopfer 1997, 1998; Hejnowicz et al. 2000), and developmental factors (Liang et al. 1996; Granger and Cyr 2001) affect the cMT orientation with respect to cell axis. The same is true also for light (Laskowski 1990; Nick et al. 1990; Zandomeni and Schopfer 1993; Ueda and Matsuyama 2000). The resultant orientation of cMTs strongly depends on a light wavelength. Blue $(\lambda=400-500 \mathrm{~nm})$ and red $(\lambda=600-700 \mathrm{~nm})$ lights which act via separate receptors (McNellis and Deng 1995) have an 
opposite effect on the cMT orientation (Zandomeni and Schopfer 1993). The fact that these changes of cMT orientation are accompanied with changes in growth rate suggests that cMTs are affected by growth and not by light per se (Fischer and Schopfer 1997).

The preliminary studies revealed that the effect of red and blue light on the cMT orientation under the outer epidermal wall in sunflower hypocotyls is manifested in a change in frequency of oblique and longitudinal or oblique and transverse cMT orientation, rather than in a change in frequency of longitudinal and transverse orientation as it was observed in coleoptiles (Zandomeni and Schopfer 1993). Since the oblique orientation of cMT occurs in two chiral forms, Z (right-handed) and S (left-handed), the chirality of cMT orientation should be taken into account.

Chirality (left-right handedness, left-right asymmetry) is a property of a number of phenomena both in animals and plants. The control of asymmetry direction can be stochastic like in phyllotaxis of plants and in the specification of the major claw in some crustaceans; or genetic like in the shell coiling direction in some snail (Palmer 2004). Most of twining plants also show fixed handedness; hop (Нитиlus) and honeysuckle (Lonicera) form left-handed helices as they climb, while Convolvulus is right-handed (Hashimoto 2002). Wild-type Arabidopsis organs do not twist. However, twisting occurs in some mutants and transgenic plants. The spiral (spr) mutants exhibit consistent righthandedly twisted organs like roots and hypocotyls due to their right-handed helical growth (Furutani et al. 2000). In the present paper the term spiral instead helical growth will be used according to Lloyd and Chan (2002). cMTs in elongating epidermal cells of these roots are arranged in left-handed helical arrays (Furutani et al. 2000). The lefty mutants in turn display left-handedly twisted organs, while their cMT arrays form right-handed helices (Thitamadee et al. 2002). It is assumed that the right- and left-handed oblique orientation of cMTs in epidermal cells is a principal cause of the left- and right-handed spiral growth of organs, respectively, and the resulting twist. Both spr and lefty mutations affect dynamics and stability of cMTs. SPIRAL (SPR) is a plant specific microtubule-localized protein required for anisotropic growth in cells (Nakajiama et al., 2004), whereas lefty mutations result from a single nucleotide exchanges in $\alpha$-tubulin genes (Thitamadee et al. 2002). Also in the transgenic Arabidopsis lines expressing modified $\alpha$-tubulins the cMTs form left-handed (S-form) helices, which causes right-handed spiral growth (Abe and Hashimoto 2005). Moreover, changes of cMT orientation chirality leading to changes in the direction of organ twisting, can be also due to treatment with chemicals that stabilize (taxol) or destabilize (propyzamide) cMTs (Furutani et al. 2000).

In the present study the orientation of parallel cMT arrays under the outer wall of sunflower hypocotyl epidermis was investigated in different irradiation. The cMT orientation is not uniform along hypocotyl; it changes basipetally: from transverse to oblique and then to longitudinal (Hejnowicz 2005). The present results show that when the orientation is oblique, the Z-form chirality dominates in the majority of examined hypocotyls kept in the darkness. Thus, the effect of blue and red light on the cMT orientation with a special regard its chirality form was studied.

\section{MATERIAL AND METHODS}

\section{Plant material and experimental treatment}

Sunflower (Helianthus annuus L. cv. Wielkopolski) achenes were immersed in $5 \%$ hydrogen peroxide for $0.5 \mathrm{~h}$, rinsed in water for $1 \mathrm{~h}$, planted in moist soil in plastic containers, and grown in darkness at $25 \pm 2^{\circ} \mathrm{C}$ for $6-7$ days. Straight hypocotyls, 6-7 cm, long were used for further experiments.

The orientation of cMT arrays was studied under the outer epidermal cell walls of hypocotyls. To study the effect of blue and red light on the cMTs, the hypocotyls were irradiated with blue $\left(\lambda=450 \mathrm{~nm}, 3 \mu \mathrm{E} \mathrm{m} \mathrm{m}^{-2} \mathrm{~s}^{-1}\right)$ or red $(\lambda=650$ $\left.\mathrm{nm}, 25 \mu \mathrm{E} \mathrm{m}^{-2} \mathrm{~s}^{-1}\right)$ light for $1 \mathrm{~h}$. Control hypocotyls were kept in darkness. Prior to material collection the hypocotyls were marked with a waterproof marker at 15 and 45 $\mathrm{mm}$ below the cotyledonary node and the epidermal strips with 2-3 layers of underlying cortex cells $(30 \mathrm{~mm}$ long and approximately $1 \mathrm{~mm}$ width) were peeled off and fixed immediately. The material from control hypoctyls was collected in dim green safety light, and from irradiated hypocotyls in blue or red light. Each experimental treatment was repeated 4-6 times.

\section{Visualization of microtubule bundles and quantification of their orientation}

Fixation and immunostaining were done as described previously (Hejnowicz 2005). The strips were observed under an epi-fluorescence microscope with a digital camera (Olympus, Japan) using 100x objective, excitation filter $470-490 \mathrm{~nm}$, and the barrier filter $-515 \mathrm{~nm}$. The cMT orientation under the outer epidermal wall was examined in three regions of the hypocotyls (each region approximately $10 \mathrm{~mm}$ long): the upper region (15-25 $\mathrm{mm}$ from the cotyledonary node), the middle region $(25-35 \mathrm{~mm})$, and the lower region $(35-45 \mathrm{~mm})$, as the cell growth and shape is known to differ between these regions (Kutschera 2000). Images of cMTs were analysed with the AnalySIS software (AnalySIS Image Processing; Soft Imaging System, $\mathrm{GmbH})$. Only the arrays in which cMTs form parallel bundles were taken into account. The angle between the bundles and the long cell axis defined by the orientation of its longitudinal walls, further denoted as $\beta$, was measured for a cell portion of a length at least twice bigger then the cell width.

At last 20 areas were analysed in each region. Twenty three hypocotyls were examined in darkness, the same number in blue and red light, giving a total of 1091-1421 cMT areas analyzed in each region. The number of examined areas differed depending on the effectiveness of immunostaining. The measured angles $(\beta)$ were grouped into 18 classes (at $10^{\circ}$ interval) and a $\beta$ frequency histogram was obtained for each region of every hypocotyl. Additionally cumulative histograms were prepared (Fig. 3) showing data calculated as mean values for all examined hypocotyls. It is assumed that when $\beta=0^{\circ}-10^{\circ}$ or $170^{\circ}-180^{\circ}$ the cMT orientation is longitudinal, while for $\beta=80^{\circ}-100^{\circ}$ it is transverse. The error bars have been estimated as the standard deviation of the mean values of the orientation frequencies in each degree interval. Statistical analysis of the occurrence of longitudinal and transverse orientation was performed using the Student's test at the 0.05 significance level. 
It has been observed that some histograms are asymmetrical, i.e. either Z-form (right-handed) or S-form (left-handed) of oblique orientations prevails. It is assumed that when $\beta=10^{\circ}-90^{\circ}$ the cMT orientation is oblique of Z-form, while for $\beta=90^{\circ}-170^{\circ}$ it is oblique of S-form. To quantify the degree to which the frequency of the occurrence the Z-form orientations exceeds or lessens the frequency of the S-form orientations, the asymmetry parameters (Q) was estimated. The parameter $\mathrm{Q}$ was calculated as the difference between the frequency (calculated as a fraction) of $\mathrm{Z}$ and $\mathrm{S}$-form orientations. Thus, when $\mathrm{Q} \neq 0$ the distribution of the cMT orientations is asymmetrical; while $\mathrm{Q}=1$ means that only oblique cMT orientations of Z-form occur. If $\mathrm{Q}=-1$ there are only oblique orientations of $S$-form. $0<\mathrm{Q}<1$ means that there is the prevalence of Z-form oblique cMT orientations, $0>\mathrm{Q}>-1$ means that $\mathrm{S}$-form oblique orientations prevail. The asymmetry parameters $\mathrm{Q}$ were qualified for appropriate region of each hypocotyl separately.

\section{RESULTS}

The orientation of parallel cMT bundles, as those shown in Figure 1a-c, in the epidermal cells was examined in hypocotyls from all of three experimental treatments (red, blue light and darkness as an control). Figure 2 shows histograms of frequency occurrence of various cMTs orientations for typical hypocotyls kept in darkness (control series, Fig. 2a-c), and irradiated with blue (Fig. 2d-f), or red (Fig. 2g-i) light for $1 \mathrm{~h}$. However, the cMT orientation histograms for hypocotyls in each series were to some extent variable. Figure 3 shows the cumulative histograms of the cMT orientations for all the 23 hypocotyls of each series. In darkness, the cMT orientation was transverse and oblique in the upper region and the histograms for this region were rather symmetrical (Fig. 2a, 3a). A tendency to decrease the frequency of transverse orientation and to increase the histogram asymmetry is apparent along the hypocotyl basipetally (Fig. 2a-c, 3a-c).

For hypocotyls irradiated with blue light (Fig. 2d-f, 3d-f) the shape of the histograms for all three regions are similar to that characteristic for the lower region of hypocotyls kept in darkness (which are considered as the control). However, statistical analysis reveals significant differences in the frequencies of the longitudinal and transverse orientations between the blue light and control experimental series. For appropriate regions of the irradiated hypocotyls the frequency of longitudinal orientation is higher, while that of transverse - lower than in control hypocotyls (Fig. 3d-f).

In the case of irradiation with red light (Fig. 2 g-i, 3 g-i) the shape of the histograms for all three regions of hypocotyls are rather similar to those characteristic for the upper region of hypocotyls kept in darkness. In that case, no statistically significant differences in frequencies of the longitudinal and transverse orientation of cMTs for appropriate regions were observed between red irradiated hypocotyls and control (Fig. 3g-i).

These results show that the cMT orientation under outer epidermal wall of sunflower hypocotyls depends on cell position along the hypocotyl. In darkness, the orientation changes basipetally from generally transverse and oblique in the upper region to oblique in the lower region. Irradiation of hypocotyls with blue light changes significantly though a small fraction the frequencies of longitudinal and transverse orientations, while in the case of red light no statistically significant effect was observed. The oblique cMT orientation is dominating in epidemis in darkness and after irradiation with blue and red light. The effect of the light concerns mainly the chirality of the oblique orientation.

Figure 3 shows that only small fraction of cMTs is strictly transversely or longitudinally oriented, while the majority of cMTs are oblique. The oblique cMT orientations occur in two chiral forms, Z (right-handed), and S (left-
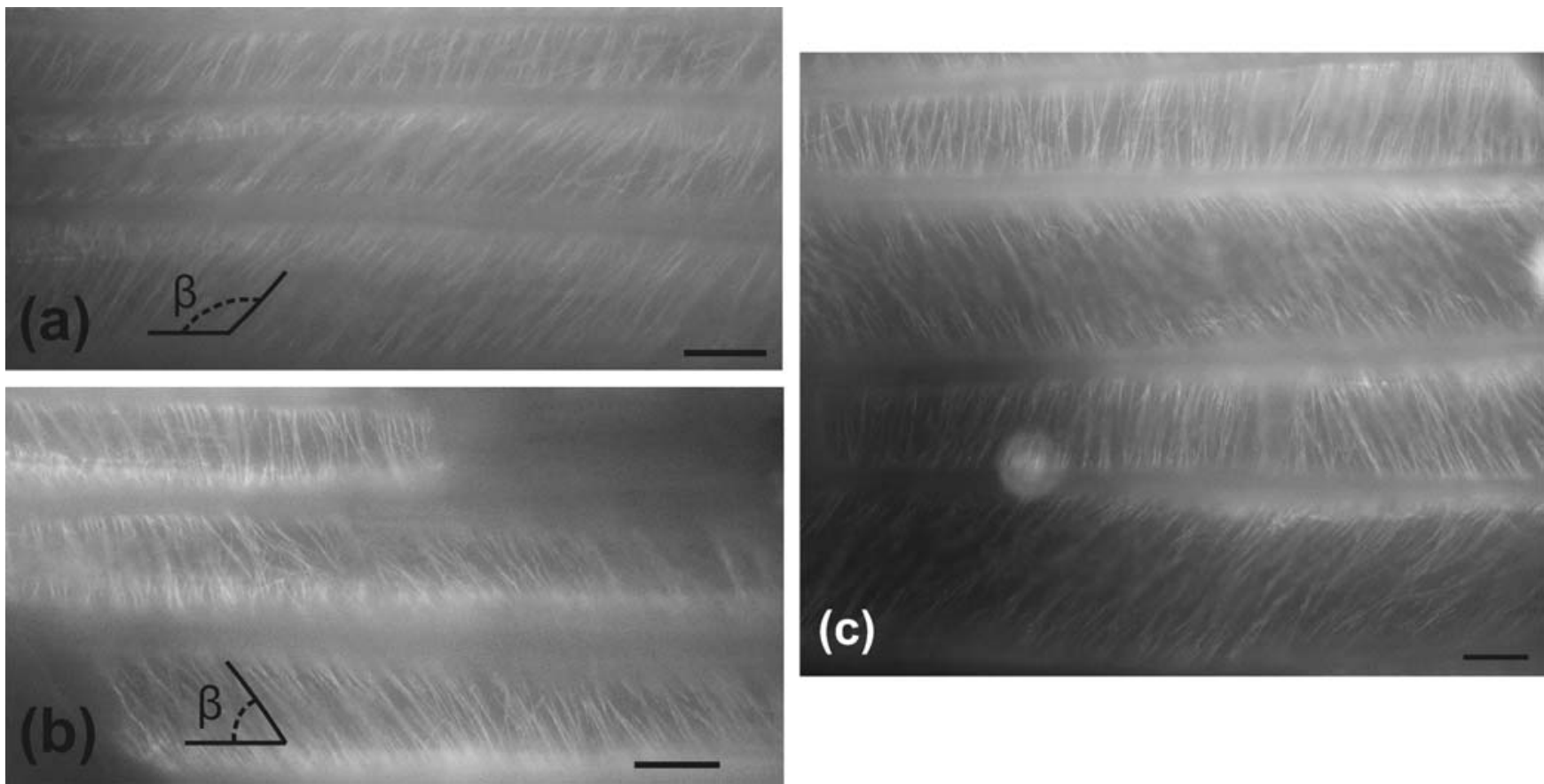

Figs 1a-c. The cMT arrays under the outer epidermal wall of sunflower hypocotyls. Nearly parallel bundles of cMTs are visible. They are inclined at particular angle $\beta$ with respect to long cell axis. Visible fragments of cells exhibit the cMT orientations of S- (a) and Z-form (b). Other fragments show mixed of S- and Z-type cMT orientations (c). Bar, $0.01 \mathrm{~mm}$. 


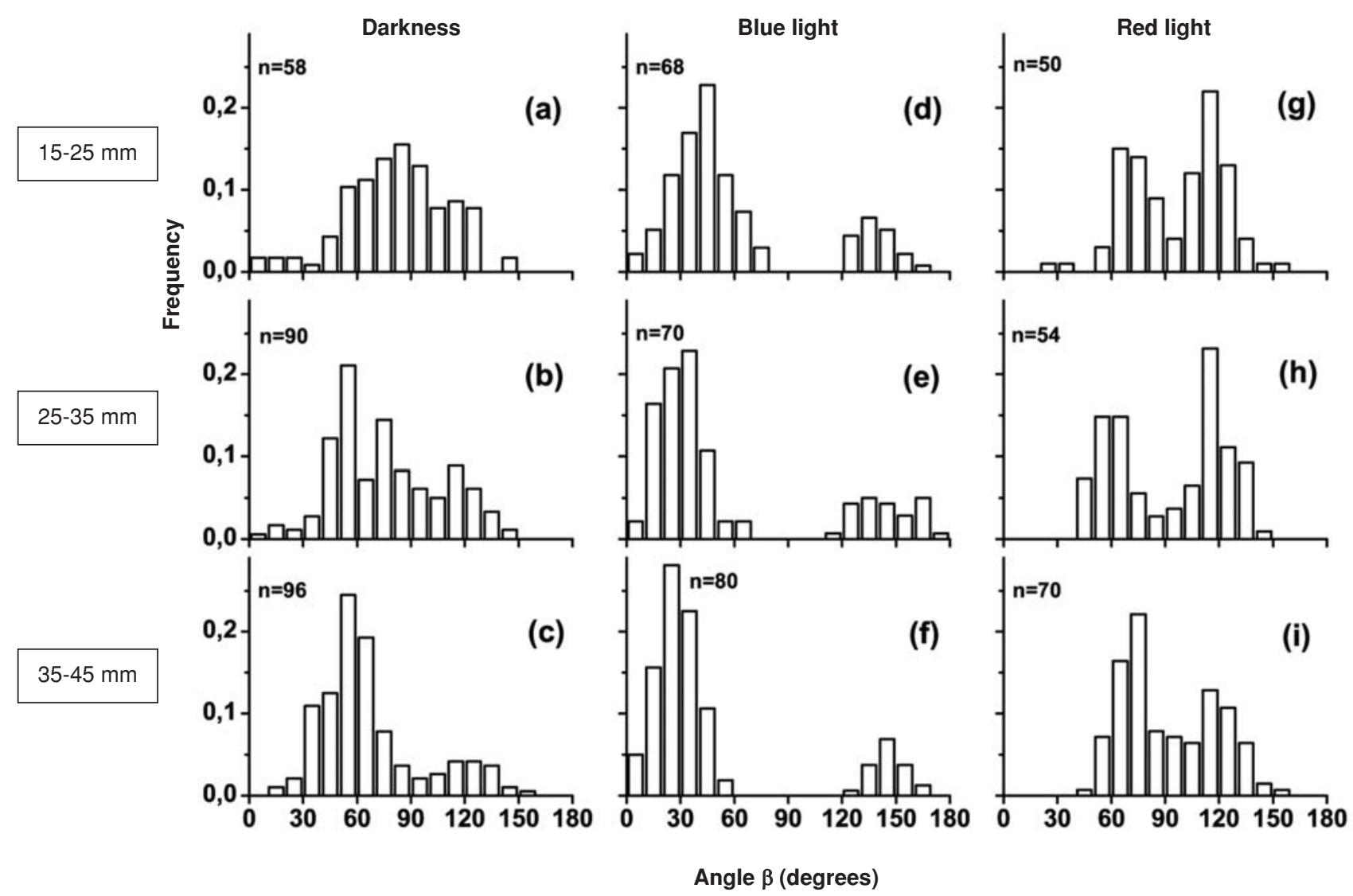

Figs 2a-i. Histograms showing the frequency distribution of the cMT orientations under the outer wall in epidermis of sunflower hypocotyl. Typical hypocotyls are shown. Histograms in one column (a-c, d-f, g-i) refer to one hypocotyl. Hypocotyls were irradiated with blue (2d-f) or red (2g-i) light. Control hypocotyls were kept in darkness $(2 \mathrm{a}-\mathrm{c})$. Numbers on the left give the distance from the cotyledonary node. $\mathrm{n}$ is the number of the cell portion examined.

-handed). If one of the forms prevails in the histogram, its shape is asymmetric, i.e. the distribution of the cMT orientation frequencies is asymmetrical. In the darkness, in the region below $25 \mathrm{~mm}$ from the cotyledonary node (i.e. in the middle and lower regions), the prevailing orientation is usually of Z-form (Fig. 2b, c, Fig. 3b, c). Such a tendency is also manifested in blue light (Fig. 2d-f, Fig. 3 d-f).

Quantitative analysis of histograms taking into account the mean values of the cMT orientation (see Buschmann et al. 2004; Abe and Hashimoto 2005) would not give satisfactory results. For example, the mean orientation in the histogram in Figure $3 \mathrm{~h}$ is $82^{\circ}$, although it is apparent that a minimum frequency is just at this angular range. Therefore, another method of histograms analysis regarding its chirality was employed.

Each cumulative histogram shown in Figure 3 shows the mean values of the frequency of cMT orientation at particular angular range computed for 23 hypocotyls. However, to study the chirality of the cMT orientation, the asymmetry of distribution of the cMT orientations must be estimated separately for each hypocotyl, since the symmetry in cumulative histograms can be due either to only symmetrical distributions of cMT orientations or equal numbers of asymmetrical distributions of opposite forms ( $\mathrm{S}$ and $\mathrm{Z}$ ) in individual histograms for a particular series. It was thus necessary to qualify the degree to which the frequency of occurrence the Z-form of cMT orientations exceeds or lessens the frequency of the S-form for all ranges in an individual histogram. The degree of histogram asymmetry was estimated with a parameter Q (see Materials and Methods). This parameter was computed for each region of every hypocotyl from a given experimental treatment. Since $Q$ values for individual hypocotyls from the particular series differed, each series was characterized by the histogram of $\mathrm{Q}$ frequency, and the mean value of $Q$ was calculated (Fig. 4).

In the upper region of hypocotyls kept in darkness (Fig. 4a) the distribution of cMT orientations frequencies in the histograms is more or less symmetrical since the majority of the $\mathrm{Q}$ values is close to 0 . However, below $25 \mathrm{~mm}$ from the cotyledonary node it changes into asymmetrical in which $\mathrm{Z}$ orientations dominate (mean value of $\mathrm{Q}$ is 0.4 and 0.5 in the middle and lower region, respectively), Figure $4 \mathrm{~b}$, c. Irradiation with blue light (Fig. 4d-f) results in asymmetrical distribution of the cMT orientation frequencies where the Z-form orientation of cMT dominate in each region of hypocotyls. Irradiation with red light has apparently different effect. The mean value of $\mathrm{Q}$ equals 0 , which indicates that red light generates rather symmetrical distribution of cMT orientations (Fig. 4g, h). Only in basal region (Fig. 4i) of some hypocotyls Z-form orientation of cMT still dominate.

\section{DISCUSSION}

The cMT orientation and its chirality with respect to the position in the organ

The present study shows that the cMT orientation in the epidermis of sunflower epidermis depends on the position along the hypocotyls. At specific position cMTs with defi- 


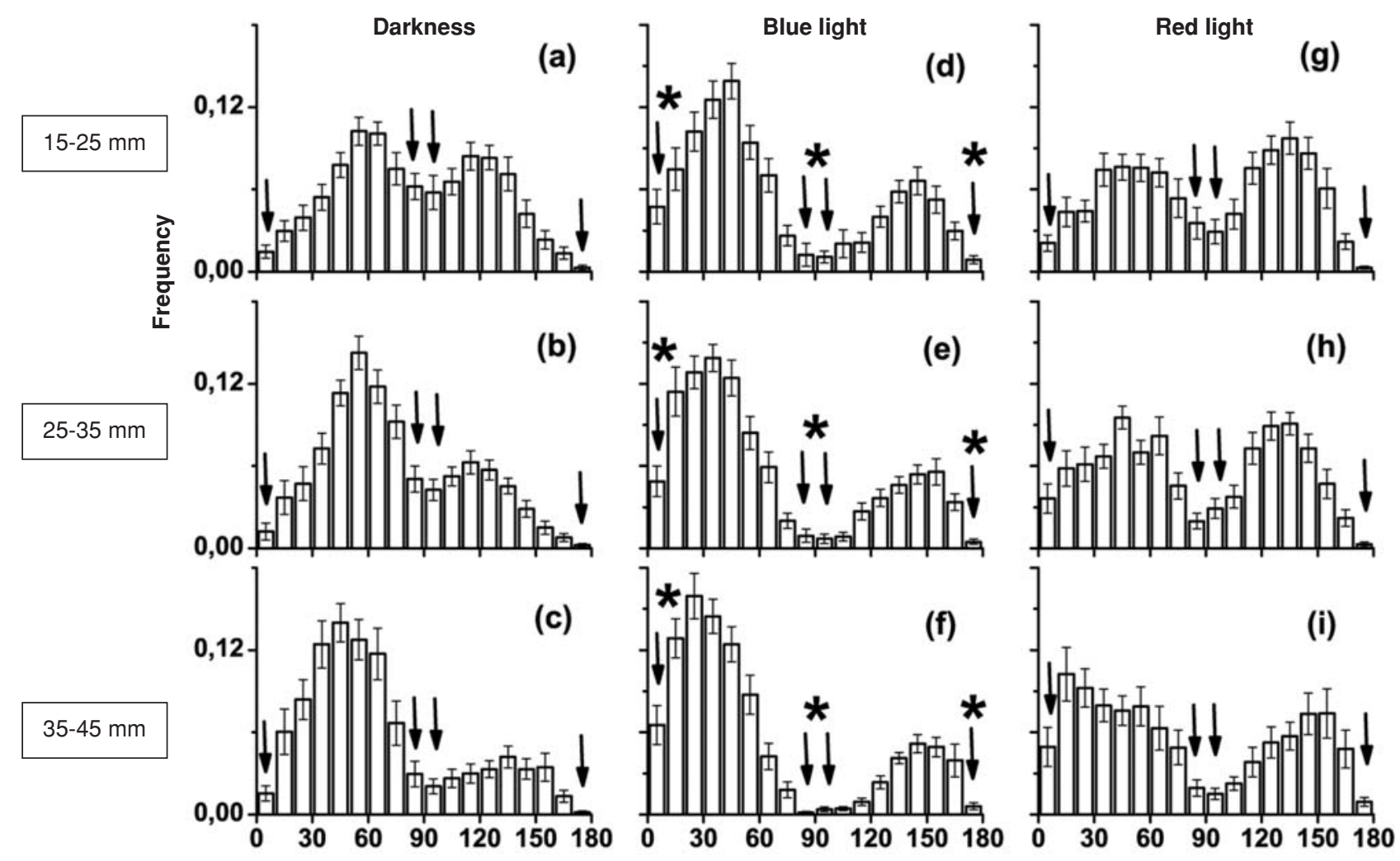

Angle $\beta$ (degrees)

Figs 3a-i. Cumulative histograms of the frequency distribution of the cMT orientations computed for 23 hypocotyls of each experimental treatment (darkness, blue and red light). Histograms in one column (3a-c, 3d-f, 3g-i) show distribution of cMT orientation along hypocotyls of a given treatment. Labeling as in Fig.2. The error bars are estimated as the standard deviation from the mean value of the orientation frequencies.

Arrows indicate angular ranges regarded as longitudinal and transverse orientation of cMTs. Statistically significant differences between frequencies of longitudinal or transverse orientations for appropriate regions in irradiated and control hypocotyls are marked with asterisks (the Student's test at the 0.05 significance level).

ned chirality dominate. In the upper hypocotyl regions cMTs are transverse and oblique of Z- as well as S-form. However, in the middle and lower regions oblique cMTs are generally of Z-form in the majority of examined hypocotyls. The positions along hypocotyl differ developmental stages of epidermal cells. The cells in the upper region are younger from those in the lower region i.e. cell divisions cease earlier in the lower then in the upper region (Kutschera 2000). There is also a gradient of growth rate along hypocotyl, the upper regions elongate faster than the lower ones (Kutschera and Köhler 1994). Therefore, the decrease in the percentage of cMTs transversely orientated and the increase in the percentage of obliquely orientated of Zform can be interpreted as a developmental change occurring in situ in darkness.

Developmental changes of cMT orientations from transverse to oblique and longitudinal have been observed in many cell types and organs like primary root, stems, or cotton fibers (Seagull 1985; Laskowski 1990; Liang et al. 1996; Baskin et al. 1999). Furthermore, it has been shown that in primary roots of maize and Arabidopsis cMT orientations have the same handedness (chirality) at a given position, and the form of cMT chirality changes along the organ (Liang et al. 1996; Baskin et al. 1999). In the cortex cell, transverse cMTs in the upper zone are followed by oblique right-handed cMTs, next by longitudinal cMTs, and finally by oblique left-handed cMTs.
However, defined chirality of cMTs in epidermis of typical stems or leaves has not been reported before. Through examination of coleoptiles and wild-type hypocotyls a domination of any form of oblique orientations was not revealed (Nick et al. 1990; Fischer and Schopfer 1998; Buschmann et al. 2004) or the chirality was not considered (Laskowski 1990; Takesue and Shibaoka 1998). Nevertheless, it is known that defined form of cMT chirality can be induced by mutations. In the elongation zone of Arabidopsis wild-type root, cMT orientations are nearly transverse, while in spr and lefty mutants cMTs form left- and righthanded arrays, respectively (Furutani et al. 2000; Thitamadee et al. 2002).

\section{The effect of blue and red light on the cMT chirality}

The present results indicate also that cMT chirality is related to their dynamics. Blue and red light are factors well known to influence the cMT orientation (Nick et al. 1990; Zandomeni and Schopfer 1993). During the present investigation it was found that irradiation with blue light induced domination of Z-form cMT orientation in the upper hypocotyl region where in the darkness neither of chiral forms was dominating. In contrast, after the irradiation with red light the domination of Z-form of chirality was less pronounced or lost, even in the lower regions. However, it cannot be distinguished whether the light affects the cMTs per se or through resulting growth changes. The fact 


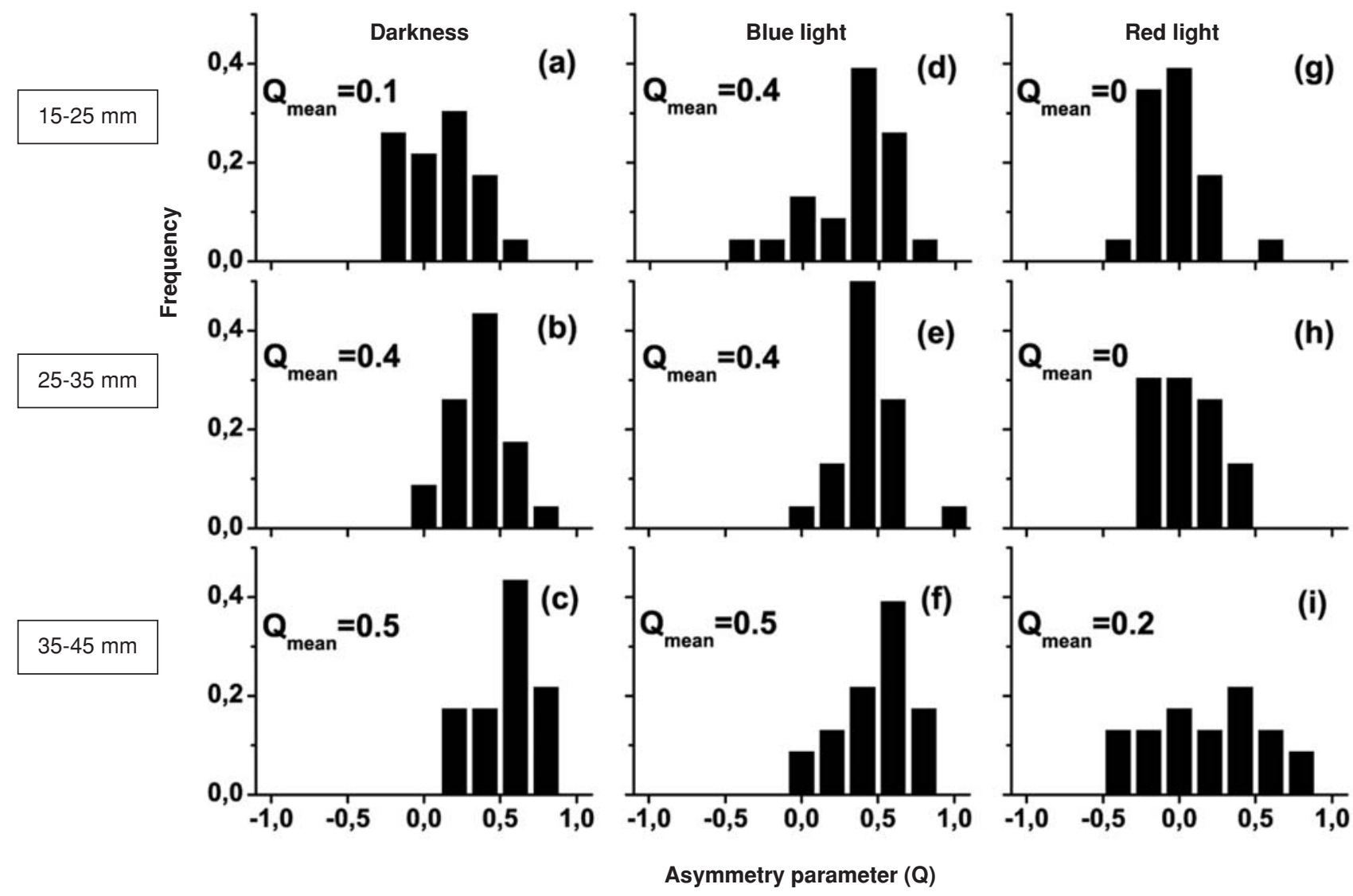

Figs 4a-i. Frequencies of occurrence of various asymmetry parameter values (Q) in hypocotyls kept in darkness (4a-c) and after irradiation with blue (4d-f) and red (4g-i) light. Each histograms is based on 23 histograms of angular frequency distribution. The mean parameter value $\mathrm{Q}_{\mathrm{mean}}$ is given for each region of hypocotyl.

that blue light generates cMT orientation that is similar to that characterizing the lower region in control can be interpreted as shifting of developmental process forward. In the case of red light, in turn, the cMT orientation is similar to that characterizing the upper region in control, which indicates the shift of developmental changes backward. These opposite effects of blue and red light are consistent with the known effect of blue and red light on cell elongation and orientation of cMTs in coleoptiles. There, one-hour irradiation with red light stimulates elongation and causes transverse orientation of cMTs, while blue light decreases elongation and causes longitudinal cMT orientation (Zandomeni and Schopfer 1993).

In the epidermis of sunflower hypocotyls, examined in the present investigation, the changes in the frequency of transverse and longitudinal cMTs were not as spectacular as it was observed in pea epicotyls (Laskowski 1990) or maize coleoptiles (Nick et al. 1990; Zandomeni and Schopfer 1993). The reason for this could be the low doses of blue light used for present experiments, but the differences in anatomy and physiology between hypocotyls and coleoptiles need also to be considered. For example, leaves being the organs especially adopted to light response, are presumably characterized by the sensitivity to light different from that of stems.

\section{The consistent cMT chirality and the spiral growth}

It is believed that cMTs generally drive deposition of cellulose microfibrils (MF) in the innermost cell wall layers and thus determine the direction of cell growth (Wymer and Lloyd 1996; Baskin 2001). It was observed that mutants and transgenic plants with consistent chirality of cMT arrays have twisted organs, i.e. in such plants the epidermal cell files of roots and hypocotyls form right- or left-handed helices as a result of spiral growth (Hashimoto 2002). According to Furutani et al. (2000) the helical MF arrangement, that is a result of helical arrangement of cMT, generates the cell elongation in the direction perpendicular to the pitch of the MF arrays. Thus, right- and left-handed helical orientations of cMT are expected to lead to left- and right-handed spiral growth, respectively. This idea is supported by the fact that helical cMT arrays were observed before twisting of epidermal cell files (Buschmann et al. 2004; Thitamadee et al. 2002). Lloyd and Chan (2002) proposed a model for this process, in which a wall with a right-handed helical construction (a cell with right-handed cellulose MFs and cMTs), unwind in the opposite direction as it is stretched (left-handed spiral growth).

In the sunflower hypocotyls there is no clearly manifested twisting. However, occasionally it was observed that in some hypocotyls, especially in their lower region, the epidermis formed slightly pronounced left-handed (S-form) helices (in approximately 5-20\% hypocotyls); while only few formed right-handed (Z-form) helices (data unpublished). Interestingly, the longer the hypocotyls, the more frequently the helical epidermis was observed. Since the Z-form of cMT orientation dominates in the lower region of sunflower hypocotyls it is possible that there is a relationship between consistent cMT chirality and the twisting, although it seems to be not as clear as in the transgenic Ara- 
bidopsis and its mutants mentioned above. Whereas the majority of hypocotyls have right-handed oblique cMTs, left-handed epidermis was observed only in the minority of cases.

\section{ACKNOWLEDGEMENTS}

I thank Zygmunt Hejnowicz for critical comments; I am also grateful to Dorota Kwiatkowska for help in preparing this manuscript.

\section{LITERATURE CITED}

ABE T., HASHIMOTO T. 2005. Altered microtubule dynamics by expression of modified á-tubulin protein causes right-handed helical growth in transgenic Arabidopsis plants. Plant J. 43: 191-204.

BASKIN T.I., MEEKES H.T.H.M., LIANG B.M., SHARP R.E. 1999. Regulation of growth anisotropy in well-watered and water-stressed maize roots. II. Role of cortical microtubules and cellulose microfibrils. Plant Physiol. 119: 681-692.

BASKIN T.I. 2001. On the alignment of cellulose microfibrils by cortical microtubules: a review and a model. Protoplasma 215: 150-171.

BLACKMAN L.M., OVERALL R.L. 1995. Electric fields affect the orientation of cortical microtubules and cell expansion in pea callus. Protoplasma 189: 156-266.

BUSCHMANN H., FABRI C.O., HAUPTMANN M., HUTZER P., LAUX T., LLOYD C.W., SCHÄFFNER A.R. 2004. Helical growth of the Arabidopsis mutant tortifolia1 reveals a plant-specific microtubule-associated protein. Curr. Biol. 14: $1515-1521$

EMONS A.M.C., DERKSEN J., SASSEN M.M.A. 1992. Do microtubules orient plant cell wall microfibrils? Physiol. Plant. 84: 486-493.

FISCHER K., SCHOPFER P. 1997. Interaction of auxin, light, and mechanical stress in orienting microtubules in relation to tropic curvature in the epidermis of maize coleoptiles. Protoplasma 196: 108-116.

FISCHER K., SCHOPFER P. 1998. Physical strain-mediated microtubule reorientation in the epidermis of gravitropically or phototropically stimulated maize coleoptiles. Plant J. 15: 119-123.

FURUTANI I., WATANABE Y., PRIETO R., MASUKAWA M., SUZUKI K., NAOI K., THITAMADEE S., SHIKANAI T., HASHIMOTO T. 2000. The SPIRAL genes are required for directional control of cell elongation in Arabidopsis thaliana. Development 127: 4443-4453.

GRANGER C.L., CYR R.J. 2001. Spatiotemporal relationships between growth and microtubule orientation as revealed in living root cells of Arabidopsis thaliana transformed with greenfluorescent-protein gene construct GFP-MBD. Protoplasma 216: 201-214.

HASHIMOTO T. 2002. Molecular genetic analysis of left-right handedness in plants. 357: 799-808. Phil. Trans. R. Soc. Lond. B 357: 799-808.

HEJNOWICZ Z. 2005. Autonomous changes in the orientation of cortical microtubules underlying the helicoidal cell wall of the sunflower hypocotyl epidermis: spatial variation translated into temporal changes. Protoplasma 225: 243-256.

HEJNOWICZ Z., RUSIN A., RUSIN T. 2000. Tensile tissue stress affects the orientation of cortical microtubules in the epidermis of sunflower hypocotyl. J. Plant Growth Regul. 19: 31-44.

HUSH J.M., OVERALL R.L. 1991. Electrical and mechanical fields orient cortical microtubules in higher plant tissues. Cell Biol. Int. Rep. 15: 551-560.
KETELAAR T., EMONS A.M.C. 2001. The cytoskeleton in plant cell growth: lessons from root hairs. New Phytol. 152: 409-418.

KUTSCHERA U., KÖHLER K. 1994. Cell elongation, turgor and osmotic pressure in developing sunflower hypocotyls. J. Exp. Bot. 45: 591-595.

KUTSCHERA U. 2000. Cell expansion in plant development. R. Bras. Fisiol. Veg. 12: 65-95.

LASKOWSKI M. 1990. Microtubule orientation in pea stem cells: a change in orientation follows the initiation of growth rate decline. Planta 181: 44-52.

LIANG B.M., DENNINGS A.M., SHARP R.E., BASKIN T.I. 1996. Consistent handedness of microtubule helical arrays in maize and Arabidopsis primary roots. Protoplasma 190: 8-15.

LLOYD C., CHAN J. 2002. Helical microtubule arrays and spiral growth. Plant Cell 14: 2319-2324.

MAYUMI K., SHIBAOKA H. 1996. The cyclic reorientation of cortical microtubules on walls with a crossed polylamellate structure: effects of plant hormones and an inhibitor of protein kinases on the progression of the cycle. Protoplasma 195: 112-122 .

McNELLIS T.W., DENG X.-W. 1995. Light control of seedling morphogenetic pattern. Plant Cell 7: 1749-1761.

NAKAJIMA K., FURUTANI I., TACHIMOTO H., MATSUBARA H., HASHIMOTO T. 2004. SPIRAL1 encodes a plantspecific microtubule-localized protein required for directional control of rapidly expanding Arabidopsis cells. Plant Cell 16: 1178-1190.

NICK P., BERGFELD R., SCHÄFER E., SCHOPFER P. 1990. Unilateral reorientation of microtubules at the outer epidermal wall during photo- and gravitropic curvature of maize coleoptiles and sunflower hypocotyls. Planta 181: 162-168.

PALMER A.R. 2004. Symmetry breaking and the evolution of development. Science 306: 828-833.

PAREDEZ A.R., SOMERVILLE C.R., EHRHARDT D.W. 2006. Visualization of cellulose synthase demonstrates functional association with microtubules. Nature 312: 1491-1495.

SEAGULL R.W. 1986. Changes in microtubule organization and wall microfibril orientation during in vitro cotton fiber development: an immunofluorescent study. Can. J. Bot. 64: 1373-1381.

SHIBAOKA H. 1994. Plant hormone-induced changes in the orientation of cortical microtubules. Annu. Rev. Plant Physiol. Plant Mol. Biol. 45: 527-544.

SHAW S.L., KAMYAR R., EHRHARDT D.W. 2003. Sustained microtubule treadmilling in Arabidopsis cortical arrays. Science 300: $1715-1718$.

TAKESUE K., SHIBAOKA H. 1998. The cyclic reorientation of cortical microtubules in epidermal cells of azuki bean epicotyls: the role of actin filaments in the progression of the cycle. Planta 205: 539-546.

THITAMADEE S., TUCHIHARA K., HASHIMOTO T. 2002. Microtubule basis for left-handed helical growth in Arabidopsis. Nature 417: 193-196.

TIAN G-W., SMITH D., GLÜCK S., BASKIN T.I. 2004. Higher plant cortical microtubule array analyzed in vitro in the presence of the cell wall. Cell Motil. Cytoskel. 57: 26-36.

UEDA K., MATSUYAMA T. 2000. Rearrangement of cortical microtubules from transverse to oblique or longitudinal in living cells of transgenic Arabidopsis thaliana. Protoplasma 213: 28-38.

WYMER C., LLOYD C. 1996. Dynamic microtubules: implications for cell wall patterns. Trends Plant Sci. 1: 222-228.

ZANDOMENI K., SCHOPFER P. 1993. Reorientation of microtubules at the outer epidermal wall of maize coleoptiles by phytochrome, blue-light photoreceptor, and auxin. Protoplasma 173: 103-112. 